DESY 93-085

ISSN $0418-9833$

June 1993

\title{
Searching for Supersymmetry in High-Energy Photon-Proton Scattering
}

\author{
W. Buchmüller, Z. Fodor円 \\ Deutsches Elektronen-Synchrotron DESY, Hamburg, Germany
}

\begin{abstract}
We investigate the possibility to search for supersymmetry in the scattering of protons and Compton back-scattered laser light. We evaluate the cross sections for inelastic and elastic production of wino pairs for different electron-proton c.m.s. energies. For $\sqrt{s}=1 \mathrm{TeV}$ the cross section exceeds $1 \mathrm{fb}$ for $m_{w}<190 \mathrm{GeV}$.
\end{abstract}

\footnotetext{
${ }^{1}$ Humboldt Fellow, on leave from Institute for Theoretical Physics, Eötvös University, Budapest, Hungary
} 
It has been known for some time that in the minimal supersymmetric extension of the standard model the running gauge couplings of strong, weak and electromagnetic interactions tend to meet at a unification scale $\Lambda_{G U T}$, whereas this is not the case in the ordinary non-supersymmetric standard model [1]. Furthermore, the unification scale $\Lambda_{G U T}$ is large enough in order to increase the theoretical predictions for the proton lifetime beyond the present experimental lower limit. Recent precision experiments [2] have confirmed [3] this observation. The unification of gauge couplings in the supersymmetric standard model appears even more remarkable if various experimental and theoretical uncertainties are carefully taken into account [4].

In view of this success several calculations of the mass spectrum in the supersymmetric standard model with radiative symmetry breaking have recently been carried out, where constraints from the top-quark mass and considerations on fine tuning of parameters were taken into account [5]. A generic feature of the resulting mass spectrum is the ordering

$$
m_{\tilde{\gamma}}, m_{z}, m_{w}<m_{h}, m_{\tilde{l}}<m_{\tilde{q}}<m_{\tilde{g}}
$$

for the mass of photino $(\tilde{\gamma})$, zino $(z)$, $\operatorname{winos}(w)$, higgsinos $(h)$, scalar leptons $(\tilde{l})$, scalar quarks $(\tilde{q})$ and gluino $(\tilde{g})$. The masses of the light gauginos $\lambda, z$ and $w$ are typically of order $100 \mathrm{GeV}$ [0].

What are the prospects to discover light gauginos in the $100 \mathrm{GeV}$ mass range at present and future colliders? In the near future LEP II offers the best chances. Here it will be possible to discover winos with masses almost up to the electron beam energy, i.e. $100 \mathrm{GeV}$. For $e^{+} e^{-}$machines with higher c.m.s. energies the discovery limit essentially scales with the beam energy. Due to lower production cross sections and higher background rates the discovery of electroweak gauginos is difficult at hadron colliders.

Recently, in connection with linear $e^{+} e^{-}$colliders, the interesting possibility has been discussed to turn an electron beam into a hard photon beam by means of Compton back-scattered laser light, and intensive theoretical work has already been carried out in order to clarify the physics potential of $e \gamma$ and $\gamma \gamma$ machines 6 . 
Hence, one may also consider the discovery potential of high-energy collisions between photons and protons. The protons are available at present storage rings and the photons could be generated by use of laser light, back scattered from electrons of a linear collider. 4 For instance, with an electron beam of $250 \mathrm{GeV}$ and a $1 \mathrm{TeV}$ proton beam one would reach a c.m.s. energy of $1 \mathrm{TeV}$. Since high energy protons yield a considerable flux of high energy photons, the resulting two-photon subprocess gives a sizeable cross section for wino pair production (cf. fig.1).

Let us consider this process in more detail. The low energy effective lagrangian for photons, W-bosons, winos and the lightest neutralino $(\lambda)$ reads

$$
\begin{aligned}
\mathcal{L} & =-\frac{1}{4} F_{\mu \nu} F^{\mu \nu}-\frac{1}{2} W_{\mu \nu}^{+} W^{-\mu \nu}+m_{W}^{2} W_{\mu}^{+} W^{-\mu} \\
& +\bar{w}\left(i \gamma^{\mu} D_{\mu}-m_{w}\right) w+\frac{1}{2} \bar{\lambda}\left(i \gamma^{\mu} \partial_{\mu}-m_{\lambda}\right) \lambda \\
& -\frac{e}{2 \sqrt{2} \sin \theta_{W}}\left(W_{\mu}^{+} \bar{\lambda} \gamma^{\mu}\left(v+a \gamma_{5}\right) w+W_{\mu}^{-} \bar{w} \gamma^{\mu}\left(v+a \gamma_{5}\right) \lambda\right),
\end{aligned}
$$

where $F_{\mu \nu}=\partial_{\mu} A_{\nu}-\partial_{\nu} A_{\mu}, W_{\mu \nu}^{-}=D_{\mu} W_{\nu}^{-}-D_{\nu} W_{\mu}^{-}$and $D_{\mu}=\partial_{\mu}-i e A_{\mu}$; $\theta_{W}$ is the weak angle, $e$ is the electric charge, $v$ and $a$ are numbers of order unity which depend on details of the chargino and neutralino mass matrices. $w$ is a Dirac fermion with the same electric charge as the $W$-boson and $\lambda$ is a Majorana fermion. For a mass spectrum satisfying eq. (2) the dominant wino decays are $w^{-} \rightarrow W^{-*} \lambda \rightarrow q_{1} \bar{q}_{2} \lambda$ and $w^{-} \rightarrow W^{-*} \lambda \rightarrow l^{-} \bar{\nu} \lambda$. For small scalar lepton masses also the decays $w^{-} \rightarrow \tilde{l}^{-*} \bar{\nu} \rightarrow l^{-} \bar{\nu} \lambda$ and $w^{-} \rightarrow l^{-} \tilde{\bar{\nu}} \rightarrow l^{-} \bar{\nu} \lambda$ have to be considered.

The total cross section for wino pair production in electron proton scattering is given by

$$
\sigma_{t o t}^{w}(s)=\int_{0}^{0.83} d x_{1} \int_{0}^{1} d x_{2} f_{\gamma \mid e}^{L A S E R}\left(x_{1}\right) f_{\gamma \mid p}\left(x_{2}\right) \sigma_{\gamma \gamma}^{w}\left(x_{1} x_{2} s\right)
$$

where $\sigma_{\gamma \gamma}^{w}$ is the wino pair production cross section in $\gamma \gamma$ scattering, $f_{\gamma \mid e}^{L A S E R}(x)$

\footnotetext{
${ }^{2}$ The question, which luminosity one can reach in high-energy photon-proton collisions, still has to be investigated.
} 
is the spectrum of the Compton back-scattered laser photons and $f_{\gamma \mid p}(x)$ is the spectrum of photons radiated from the proton.

The laser photon spectrum has been calculated by I.F. Ginzburg et al. [0]. Contrary to the Weizsäcker-Williams spectrum it is hard [0, 8]

$$
f_{\gamma \mid e}^{L A S E R}(x)=2 \frac{(1+Y)^{2}}{(1-x)^{2}} \times
$$

$\frac{2 Y^{2}-4 Y(1+Y) x+\left(4+4 Y+3 Y^{2}\right) x^{2}-Y^{2} x^{3}}{Y\left(16+32 Y+18 Y^{2}+Y^{3}\right)-2\left(8+20 Y+15 Y^{2}+2 Y^{3}-Y^{4}\right) \ln (1+Y)}$,

and peaks at the maximum value of $x_{\max }=Y /(1+Y)=0.83$ for the optimized value of the parameter $Y=4.82$. The photon spectrum $f_{\gamma \mid p}(x)$ has an inelastic component which is obtained by summing the WeizsäckerWilliams spectra of the quarks inside the proton in the case of inelastic proton scattering,

$$
f_{\gamma \mid p}^{\text {inel }}(x)=\int_{0}^{1} d x_{1} \int_{0}^{1} d x_{2} \sum_{q} f_{\gamma \mid q}\left(x_{1}\right) f_{q}\left(x_{2}, Q^{2}\right) \delta\left(x-x_{1} x_{2}\right)
$$

here $f_{q}\left(x, Q^{2}\right)$ are the parton densities and $f_{\gamma \mid q}(x)$ is the Weizsäcker-Williams spectrum

$$
f_{\gamma \mid q}(x)=e_{q}^{2} \frac{\alpha}{2 \pi} \frac{1+(1-x)^{2}}{x} \ln \frac{t_{\text {max }}}{t_{c u t}},
$$

where $t_{\max }$ and $t_{c u t}$ are characteristic maximal and minimal momentum transfers. Following [9], we choose $t_{\max }=\hat{s}-4 m_{w}^{2}$ for wino pair production, where $\hat{s}$ is the photon-quark c.m.s. energy, and $t_{c u t}=1 \mathrm{GeV}^{2}$. Furthermore we use the Duke-Owens parton densities set 1, with $Q=100 \mathrm{GeV} 10$.

Several years ago, Drees and Zeppenfeld have shown for the case of scalar electron-photino production in $e^{-} p$ scattering that the cross section for the elastic process $e^{-} p \rightarrow \tilde{e} \lambda p$ and the inelastic process $e^{-} p \rightarrow \tilde{e} \lambda p$ are of the same size [11]. This is very interesting, especially since the elastic process yields a very clean final state. The elastic cross section can be evaluated using a modified Weizsäcker-Williams approximation. An accurate expression for 
the corresponding photon spectrum has been derived by Kniehl [12]:

$$
f_{\gamma \mid p}^{e l}(x)=-\frac{\alpha}{2 \pi} x \int_{-\infty}^{t_{0}} \frac{d t}{t}\left(2\left[\frac{1}{x}\left(\frac{1}{x}-1\right)+\frac{m_{p}^{2}}{t}\right] H_{1}(t)+H_{2}(t)\right),
$$

where $m_{p}$ is the proton mass and $t_{0}=-m_{p}^{2} x /(1-x) \cdot H_{1}$ and $H_{2}$ are combinations of the electric and magnetic form factors of the proton, $G_{E}(t)=$ $\left(1-t / 0.71 \mathrm{GeV}^{2}\right)^{-2}$ and $G_{M}(t)=2.79 G_{E}(t)$,

$$
\begin{aligned}
& H_{1}(t)=\frac{G_{E}^{2}(t)-\left(t / 4 m^{2}\right) G_{M}^{2}(t)}{1-t / 4 m^{2}}, \\
& H_{2}(t)=G_{M}^{2}(t)
\end{aligned}
$$

From eqs. $(\overline{7}, 8)$ one can obtain the explicit expression for $f_{\gamma \mid p}^{e l}(x)$ which can be found in ref [12].

In order to obtain the wino pair production cross section (3) we finally need the cross section of the two-photon subprocess [13],

$$
\begin{aligned}
& \sigma_{\gamma \gamma}^{w}(s)= \\
& 4 \pi \alpha^{2}\left(\left(1+\frac{4 m^{2}}{s}-\frac{8 m^{4}}{s^{2}}\right) \ln \left(\frac{1+\beta}{1-\beta}\right)-\beta\left(1+\frac{4 m^{2}}{s}\right)\right) s^{-1},
\end{aligned}
$$

where $s>4 m_{w}^{2}$ and $\beta=\left(1-4 m_{w}^{2} / s\right)^{1 / 2}$ is the wino velocity. We shall also need the corresponding cross section for the production of $W$-boson pairs [14],

$$
\begin{aligned}
& \sigma_{\gamma \gamma}^{W}(s)= \\
& 8 \pi \alpha^{2} \beta\left(1+\frac{3 m^{2}}{4 s}+\frac{3 m^{4}}{s^{2}}-3 m^{4}\left(1-\frac{2 m^{2}}{s}\right) \ln \left(\frac{1+\beta}{1-\beta}\right) s^{-2} \beta^{-1}\right) m^{-2},
\end{aligned}
$$

which, for $m_{W}<m_{w}$, is always larger than the production cross section for winos.

From eqs. (3)-(9) one now obtains the elastic and inelastic wino pair production cross sections. The results are shown in fig. 2 for three different 
electron-proton c.m.s energies, $\sqrt{s}=314,450,1000 \mathrm{GeV}$. Note, that the elastic cross section is slightly larger than the inelastic one. The total cross sections are plotted on fig. 3. At the HERA energy of $314 \mathrm{GeV}$ the cross section is $10 \mathrm{fb}$ for a wino mass of $60 \mathrm{GeV}$, which is significantly below the reach of LEP II. At $1 \mathrm{TeV}$ c.m.s. energy the production cross section is larger than $1 \mathrm{fb}$ for $m_{w}<190 \mathrm{GeV}$, which corresponds to the reach of a linear collider with c.m.s. energy of about $400 \mathrm{GeV}$.

Winos decay predominantly into 2 jets and a neutralino (cf. fig. 1). Wino pair production therefore leads to final states with 4 jets, missing energy and the proton remnant, a clean signature for supersymmetry. For the leptonic wino decay, $w^{-} \rightarrow l^{-} \bar{\nu} \lambda$, the final state would contain a charged lepton pair $l^{+} l^{-}$, missing energy and the proton remnant. In this case the background from $W$-boson pair production with leptonic $W$-boson decay is important. The elastic and inelastic $W$-boson cross sections can be obtained from eqs. (3)-(8), (10) and are shown in fig.4.

We conclude that high energy photon-proton scattering would allow to search for super-particles in a very interesting mass range, which raises the question of the general physics potential of a photon-proton collider.

We thank D. Zeppenfeld and P. Zerwas for helpful discussions. Z.F. acknowledges partial support from Hung. Sci. Grant under Contract No. OTKA-F1041. 


\section{References}

[1] U. Amaldi et al., Phys. Rev. D36 (1987) 1385

[2] LEP collaborations, Phys. Lett. B276 (1992) 247

[3] J. Ellis, S. Kelley and D.V. Nanopoulos, Phys. Lett. B249 (1990) 441;

U. Amaldi, W. de Boer and H. Fürstenau, Phys.Lett. B260 (1991) 447;

P. Langacker and M. Luo, Phys. Rev. D44 (1991) 817

[4] P. Langacker and N. Polonsky, preprint UPR-0513T (1992)

[5] G. G. Ross and R. G. Roberts, Nucl. Phys. B377 (1992) 571; R. Arnowitt and P. Nath, Phys. Rev. Lett. 69 (1992) 725 S. Kelley et al. Nucl. Phys. B398 (1993) 3

[6] V. Telnov, in Physics and experiments with Linear Colliders, eds. R. Orava et al. (World Scientific, Singapore, 1992) p. 739

[7] I.F. Ginzburg, G.L. Kotkin, V.G. Serbo and V.I. Telnov, Nucl. Instr. and Methods 205 (1983) 47

[8] K. Hagiwara, I. Watanabe and P. Zerwas, Phys. Lett. B278 (1992) 187

[9] G. Altarelli, G. Martinelli, B. Mele and R. Rückl, Nucl. Phys. B262 (1985) 204

[10] D. Duke and J. F. Owens, Phys. Rev. D30 (1984) 49

[11] M. Drees and D. Zeppenfeld, Phys. Rev. D39 (1989) 2536

[12] B. A. Kniehl, Phys. Lett. B254 (1991) 267

[13] S.J. Brodsky T. Kinoshita and H. Terazawa, Phys. Rev. D4 (1971) 1532

[14] I.F. Ginzburg et al. Nucl. Phys. B228 (1983) 285 


\section{Figure caption}

1. Feynman diagram of the wino pair production and dominant decay in photon-proton collision.

2. Cross sections of the elastic (solid line) and inelastic (dashed line) wino pair productions at different c.m.s. energies.

3. Total cross section of the wino pair productions at different c.m.s. energies.

4. The $W^{+} W^{-}$production cross sections at the corresponding energies (short dashed: elastic contribution, long dashed: inelastic contribution, solid line: sum). 